\title{
Desastres naturales y responsabilidad civil. Identificación de los desafíos que presenta esta categoría de hechos dañinos
}

“...tiene este Chile florido algo de Sísifo, ya que como él, parece condenado a que se le venga abajo cien veces, lo que cien veces elevó”.

(Ortega y Gasset, discurso en el

Parlamento chileno, 1928).

\section{Lilian San Martín Neira*}

\section{RESUMEN}

Este trabajo presenta varias reflexiones necesarias para encuadrar correctamente a los desastres naturales al interior del sistema de responsabilidad civil chileno, como un paso previo e indispensable a la posible configuración de una subcategoría de la responsabilidad civil, llamada responsabilidad civil por desastres naturales. Con tal objetivo, se describen los aspectos que caracterizan a los desastres naturales como hipótesis de riesgos y hechos dañinos, poniendo en evidencia las dificultades que necesariamente deben sortearse si se quiere tener un sistema de responsabilidad civil coherente. En particular, se destacan los problemas asociados a la prueba del nexo causal y a la extensión de la indemnización, así como el posible ejercicio de la acción de daño contingente para prevenir la ocurrencia de desastres, identificando los problemas que deben resolverse para que esta acción prospere.

Responsabilidad civil por desastres naturales - causalidad - daño contingente

\section{Natural desasters and civil liability. Detection of the challenges presented by this category of harmful facts}

\section{Abstract}

This paper presents a series of thoughts needed to fit properly natural disasters into the Chilean system of civil liability, as a prior and essential step to the possible configuration of a subcategory of the civil liability, called civil liability for natural disasters. To this goal, it describes

* Licenciada en Ciencias Jurídicas y Sociales, Universidad de Concepción. Máster y Doctor en Sistema Jurídico Romanista, Universidad de Roma "Tor Vergata”, Italia. Profesora de Derecho Civil, Universidad Alberto Hurtado. Correo electrónico 1sanmar@uahurtado.cl.

Este artículo forma parte del proyecto Fondecyt N ${ }^{\circ} 1170686$.

Artículo recibido el 3 de enero de 2019 y aceptado para su publicación en este número el 1 de agosto de 2019 
the aspects that characterize disasters as hypothesis of risks and harmful acts, highlighting the complications that must necessarily be removed if you want a coherent system of civil liability. In particular, it highlights the problems associated with the test of causation and the extent of the compensation, as well as the possible exercise of the action for contingent damages, to prevent the occurrence of disasters, identifying the problems that need to be resolved so that such an action is to prosper.

Civil liability for natural disaster - causation - contingent damages

\section{INTRODUCCIÓN}

C omo es sabido, Chile es un país altamente expuesto a fenómenos naturales extremos, como terremotos, tsunamis, erupciones volcánicas, inundaciones, incendios forestales. Esta circunstancia ha determinado que a lo largo de su historia haya debido convivir con una variada gama de desastres naturales ${ }^{1}$, que eran asumidos como "infortunios" o "fatalidad" por quienes los sufrían y, por consiguiente, en general no daban origen a demandas de responsabilidad civil. En los últimos años, especialmente a raíz del terremoto de 27 de febrero de 2010 (en adelante 27F), esta situación ha cambiado, las víctimas han ejercido demandas en contra de quienes consideran "responsables" del daño sufrido, más allá de que en los hechos el factor que lo generó haya sido un fenómeno natural ${ }^{2}$.

Así, está surgiendo un nuevo nicho de responsabilidad civil, que requiere ser analizado, con el fin de encuadrarlo correctamente. Para cumplir esta finalidad, resulta necesario individualizar las particularidades de los desastres naturales como especiales categorías de hechos dañinos, identificando los desafíos que ellos generan en relación con los requisitos generales de la responsabilidad civil. Este es precisamente el objetivo de este texto, el que reflexiona en torno a la identificación de algunos aspectos problemáticos presentados por los desastres naturales como hechos dañinos. El análisis se realiza con miras al posible desarrollo de una teoría de la responsabilidad civil derivada de desastres naturales, como ya ha ocurrido con otras categorías de daños: $v$ gr. accidentes laborales, accidentes automovilísticos, negligencia médica, entre otros.

Salvo escasas referencias jurisprudenciales, el desarrollo de la exposición estará centrado en las características de los desastres naturales como categorías-riesgos y los problemas es necesario superar para su correcto encuadramiento en el sistema de responsabilidad

\footnotetext{
${ }^{1}$ Acerca del particular, vid. Palacios, 2016, pp. 41 ss.; Onetto, 2017, pp. 37 ss.
}

${ }^{2}$ El hecho de que hasta el terremoto del 2010 la sociedad chilena considerara los desastres naturales, y en particular a los terremotos, como una "fatalidad", se revela también en la literatura que describe el carácter del chileno en relación con la sismicidad del territorio nacional, utilizando expresiones como "estoicismo" y "resignación”. "De hecho, el adjetivo "estoico" se repetía con frecuencia, en alusión al destino de enfrentar una y otra vez la pérdida de posesiones materiales en el caso de Chile, por la acción imprevista de la naturaleza, y no alterarse mayormente por ello”. Cfr. Silva y Riquelme, 2018, p. 72. Una situación diametralmente opuesta se observa luego del $27 \mathrm{~F}$, en que el "evento natural" pasa a segundo plano, tomando primacía las supuestas "ausencia de políticas públicas y la negligencia de las autoridades”. Cfr. UGARTE et al., 2014, p. 138. 
civil chileno. De esta manera, se pretende establecer las bases teóricas para una futura discusión acerca del argumento, que tome partido respecto de las soluciones más idóneas y que, eventualmente, realice un análisis crítico de la copiosa jurisprudencia nacional actualmente existente en la materia.

\section{LOS DESASTRES NATURALES FRENTE A LA NECESIDAD}

\section{DE DISTRIBUIR EL COSTO DEL ACCIDENTE}

Según la Cepal, los desastres naturales "son consecuencia de fenómenos naturales desencadenantes de procesos que provocan daños físicos y pérdidas de vidas humanas y de capital, al tiempo que alteran la vida de comunidades y personas, y la actividad económica de los territorios afectados"3. Como se desprende de esta definición, la ocurrencia de desastres naturales implica la pérdida de vidas humanas y de recursos económicos, en una relación directamente proporcional a la magnitud del desastre, esto determina que algunos desastres afecten de manera significativa el crecimiento y desarrollo del país, lo que ha significado una creciente preocupación por estos fenómenos desde una perspectiva económico-social, que involucra principalmente a la actividad estatal y, en consecuencia, suele ser mirada desde el Derecho público, pero al Derecho privado también corresponde un rol en la materia.

En efecto, si bien en el imaginario colectivo la prevención y gestión de desastres son tareas estatales, lo cierto es que ellas involucran también a los particulares. De lo anterior resulta que los estudiosos del Derecho privado -como rama del Derecho destinada fundamentalmente a regular las relaciones entre particulares- también estamos llamados a revisar las instituciones y herramientas con que esta disciplina puede aportar a la prevención y gestión de los desastres ${ }^{4}$.

Ahora bien, cuando se piensa en los desastres naturales desde la perspectiva del Derecho privado, inmediatamente se asocia al desastre con el daño que genera y con quién es el jurídicamente llamado a soportarlo. Siguiendo a Calabresi ${ }^{5}$, es admisible preguntarse ¿quién soporta el costo del accidente llamado desastre natural? En efecto, en la perspectiva iusprivatista, el principal problema que acarrean los desastres naturales son las pérdidas (daño emergente y lucro cesante) derivadas de la destrucción de los bienes, así como el daño moral derivado de la afectación de la vida, salud e integridad física y psíquica de las comunidades damnificadas, lo que lleva a preguntarse quién debe

${ }^{3}$ Cepal, 2014, p. 17. En Chile, aunque de manera más general y mirando exclusivamente al diseño de políticas públicas (lo que explica las altas exigencias que impone la definición), los desastres han sido definidos en el Anexo 2 del Decreto 1512 del Ministerio del Interior, como "una seria interrupción en el funcionamiento de una comunidad o sociedad que ocasiona una gran cantidad de muertes al igual que pérdidas e impactos materiales, económicos y ambientales que exceden la capacidad de la comunidad o la sociedad afectada para hacer frente a la situación mediante el uso de sus propios recursos".

${ }^{4}$ En algunos ordenamientos extranjeros ya es posible observar esfuerzos en este sentido, v. gr. vid. Jordano, 2000; Posner, 2004; Faure y Hartlief, 2006, Bruggeman, 2010, Lacroix, 2012.

${ }^{5}$ Calabresi, 1970 , pp. 3 ss. 
pagar por ese daño ${ }^{6}$. En definitiva, se trata de una cuestión de distribución de riesgos y el Derecho privado posee desde antiguo herramientas creadas con ese propósito.

Al efecto, es valioso recordar que, en línea de máxima, frente la verificación de un accidente que ocasione daños, teóricamente, una sociedad puede adoptar diversas posiciones, dependiendo de qué tan liberal o colectivista sea. En general, puede establecer que el autor material del daño deba siempre repararlo, socializarlo mediante seguros obligatorios o un sistema de seguridad social, o establecer que la víctima debe siempre asumir sus costos. Según afirma el mismo Calabresi, ninguna sociedad ha adoptado una fórmula pura, todas tienen una fórmula mixta ${ }^{7}$, con el predominio de una regla general en donde lo usual será que sea la víctima quien soporte el daño (casus sentit dominus), a menos que tenga fundamentos para traspasarlo a un tercero. Esto es justamente lo que ocurre con los desastres naturales: el primer llamado a soportarlo es la víctima, en cuanto son consecuencia de fenómenos naturales, a menos que pueda traspasar su costo a un tercero, lo que puede hacer por medio del mecanismo de los seguros o bien de la responsabilidad civil.

Visto de esa manera, el Derecho privado aparece más bien reactivo: se ocupa del fenómeno una vez acaecido. Sin embargo, también posee herramientas que pueden emplearse ex ante. Especialmente relevante en este sentido es la función preventiva de la responsabilidad civil, que en Chile ha sido expresamente acogida en la acción por daño contingente contemplada en el artículo 2333 CC, y que a nuestro juicio resulta útil para prevenir la ocurrencia de desastres.

En síntesis, desde la perspectiva del Derecho privado, dos son las principales herramientas útiles para la gestión y prevención de desastres: los seguros y la responsabilidad civil, esta última tanto en su función compensatoria como preventiva.

En Chile no se cuenta con seguros obligatorios para catástrofes ${ }^{8}$, en consecuencia, al margen de la asistencia social que proporcionan el Estado y algunas organizaciones no gubernamentales, cobra gran importancia la responsabilidad civil, pues es el mecanismo con el que la población obtiene indemnización?

${ }^{6}$ Esta preocupación ha estado en la mente de los estudiosos de las catástrofes, no solo naturales, sino de toda índole, y se han generado diversas publicaciones en torno al argumento, $v$. gr. FAURE, Y HARTLIEF, 2006; Bruggeman, 2010; Faure y Jing, 2012.

7 Calabresi, 1970, pp. 274 ss.; Calabresi, 1978, pp. 519-534.

${ }^{8} \mathrm{La}$ única norma que podría considerarse una excepción en la materia la constituye el artículo 36 de la Ley 19.537, que establece el seguro de incendio en materia de copropiedad inmobiliaria. Con todo, se trata de una norma de alcance muy limitado y, por lo demás, el seguro que se contrate es de carácter exclusivamente privado, sin que haya intervención estatal de ningún tipo.

${ }^{9}$ Corral, 2010, pp. 167-176; Pizarro, 2010, pp. 161-176; Revista del Abogado, No 48, abril 2010; LARA y GARCía-Huidobro, 2014, pp. 163-194. Lo mismo ocurre en otros países de la región, $v$. gr. https:// xaflag.blogspot.cl/2016/07/caso-fortuito-el-terremoto-del-16-de.html. Aunque pueda resultar criticable la instrumentalización de la responsabilidad civil para resolver problemas que son más bien de política pública, esta situación evidencia el rol público (y de justicia distributiva) que también cumple la responsabilidad, sobre el particular, vid CANE, 2014, p. 148. 


\section{LOS DESASTRES NATURALES COMO HIPÓTESIS ESPECIALES DE “HECHOS DAÑINOS”}

En cada ordenamiento jurídico existen reglas de atribución de responsabilidad que responden (o debieran responder) a una lógica de sistema, de manera que resulten coherentes entre sí, pues eso contribuye a la preciada seguridad jurídica; sin embargo, como afirma Viney, tal necesaria coherencia no implica uniformidad de tratamiento a los daños derivados de las distintas actividades desarrolladas por los actores sociales. Al contrario, el sistema debe estar construido sobre la base de unos principios generales suficientemente firmes y coherentes, que permitan dar soluciones satisfactorias y armonizadas a los problemas que resultan de situaciones que exigen un estatuto particular ${ }^{10}$. A nuestro juicio, este es precisamente el caso de los desastres naturales. Ellos constituyen situaciones extraordinarias en el contexto de la responsabilidad civil, pues los fenómenos naturales que los desencadenan están fuera del control humano, no es posible identificar un sujeto a la base del fenómeno natural. De ahí que sea necesario identificar las particularidades de los desastres naturales como fuentes de responsabilidad civil y el tratamiento jurídico que estas requieren, reflexionando acerca de los desafíos que esto conlleva para su coherente encuadramiento en el sistema general de responsabilidad civil. A tal objetivo se abocará este texto, centrándose fundamentalmente en poner en evidencia estos desafíos, en la esperanza de que ello contribuya a encuadrar de mejor manera los casos en que se discute la responsabilidad civil derivada de desastres naturales $y$, en un futuro cercano, contar con reflexiones más acabadas pertinentes al argumento, que permitan construir una rama de la responsabilidad civil llamada "responsabilidad derivada de desastres naturales", así como ha ocurrido con la "responsabilidad por accidentes del trabajo", "la responsabilidad médica", "la responsabilidad por productos defectuosos”, etc. Para ello el análisis será dividido en dos partes: (1) los desastres naturales y la responsabilidad civil en su faz compensatoria; y (2) los desastres naturales como hipótesis de daño contingente.

\section{Los desastres naturales y la responsabilidad civil en su faz compensatoria}

La llamada faz compensatoria de la responsabilidad civil supone que el daño ya ha acaecido y es necesario identificar al civilmente responsable del mismo. Al efecto, es importante recordar que, además de la culpa, que eventualmente puede faltar, los elementos esenciales de la responsabilidad son precisamente la existencia del daño y el nexo causal. Desde esta perspectiva, los desastres naturales proponen múltiples desafíos, comenzando por el hecho de que el detonante directo del daño será siempre un fenómeno natural, esto es, un suceso ajeno a la voluntad de cualquiera de los intervinientes, lo que, según se verá a continuación, representa una dificultad para el establecimiento del nexo causal, así como como para la determinación del quantum indemnizatorio.

\footnotetext{
${ }^{10}$ VINEY, 2007, pp. 125 ss.
} 
A. Los problemas de establecimiento del nexo causal: herramientas a considerar para su solución

Aunque en cierta medida predictibles, los fenómenos a la base de los desastres naturales generalmente escapan al control humano y, por lo mismo, suelen ser calificados de "fuerza mayor" o "caso fortuito"11. Ellos constituyen un "acto de Dios" (act of God); sin embargo, frecuentemente, el despliegue de su potencialidad dañina se debe a un "acto del hombre", así como la construcción de una represa, el desvío del cauce de un río, la cancelación de una alerta de tsunami, etc. En estos casos, debido a que el ámbito de protección de la responsabilidad extracontractual está delimitado por la "culpa"12, solo habrá lugar a ella en la medida en que se determine que la causa determinante del daño fue el hecho (culpable) del hombre y no el fenómeno natural. Si, por el contrario, se establece que la causa determinante fue el fenómeno natural, la víctima deberá soportar el daño: casus sentit dominus.

En consecuencia, para que haya responsabilidad, debe quedar acreditado en autos que el daño se ha producido "gracias a la intervención del demandado"13, lo que supone probar el vínculo de causalidad natural entre el hecho del demandado y el daño, por medio del test de la conditio sine qua non ${ }^{14}$. Ahora bien, ya que el respectivo fenómeno natural es sin duda una condición necesaria del daño, la prueba debe versar acerca de una de estas dos posibilidades: (i) que, a pesar del fenómeno natural, de no haber mediado la intervención del demandado, el daño no se habría producido; o (ii) que, a pesar del fenómeno natural, de haber mediado la oportuna intervención del demandado (anterior, concomitante o posterior al hecho), el daño no se habría producido. Esto supone grandes retos desde el punto de vista probatorio, por dos órdenes de razones: en primer lugar, porque implica acreditar un contrafáctico, es decir, reemplazar mentalmente una situación efectivamente acontecida con otra ideal, que no tuvo ni puede tener lugar ${ }^{15}$; en segundo lugar, porque los desastres naturales usualmente causan conmoción y crean un clima generalizado de confusión, sin que haya claridad de cómo acontecieron los hechos a $\operatorname{probar}^{16}$. Estas circunstancias dificultan la prueba de la causalidad al punto de hacerla prácticamente imposible, se produce así una "incertidumbre causal”, que deberá necesariamente ser resuelta mediante decisiones político-jurídicas ${ }^{17}$.

${ }^{11}$ Letelier, 2001, pp. 1 ss.; Letelier, 2012a, pp. 303 ss.; Letelier, 2012 b pp. 1 ss.; TApia, 2012, p. 57.

${ }^{12}$ SCHOpf, 2015, p. 923.

13 Infante, 2002, pp. 1 ss.; Baraona, 2003, pp. 366 ss.; Raikes y McBean, 2016, pp. 12-18.

${ }^{14}$ Esta demostración es un requisito mínimo para la atribución de responsabilidad, no se trata de aplicar puramente la doctrina de la equivalencia de las condiciones. De este tema, BARros, 2006, pp. 376 ss.

${ }^{15}$ Respecto de los contrafácticos en relación con los juicios causales, vid RATTi 2014, pp. 95 ss.

${ }^{16}$ Esto explica que una de las mayores dificultades que han debido enfrentar los tribunales, por ejemplo, a propósito de las demandas instauradas a raíz del 27F, haya sido precisamente el establecimiento (prueba) del nexo causal. Vid San Martín, 2013, pp. 305 ss.; Poblete, 2015, pp. 243-251; Ríos, 2017, pp. 64 ss.

${ }^{17}$ En este sentido se habla de una suerte de “juridización” de la causalidad natural, pues se establece mediante recursos que son más bien de carácter jurídico, como la substitución de la verificación (en el verdadero sentido de la palabra) causal por coeficientes de probabilidad medio-bajos; o bien por medio del 
Ciertamente, la incertidumbre causal no es un problema exclusivo de los desastres naturales, pero en estos resulta de especial gravedad, atendido el número de afectados y la ya mencionada confusión que causan en la población. De ahí que la configuración de un régimen especial de responsabilidad civil para este tipo de daños deba necesariamente considerar los expedientes con el Derecho probatorio que cuenta para enfrentar este tipo de situaciones, máxime cuando alguna doctrina ha propuesto que ciertas tipologías de casos, por su especial complejidad, admitirían mecanismos especiales para enfrentar la incertidumbre causal ${ }^{18}$.

Aclarada la razón por la que resulta relevante analizar esta cuestión en esta sede, es importante señalar que doctrinariamente los problemas de incertidumbre causal han sido enfrentados con distintos mecanismos, algunos de estos corresponden a herramientas generales del derecho probatorio, válidos para cualquier hecho controvertido, este es el caso de las presunciones y los estándares probatorios. Mientras que otros han sido diseñados precisamente para resolver problemas de incertidumbre causal en la responsabilidad civil, como es el caso de las teorías acerca de responsabilidad proporcional, pérdida de chance y aumento del riesgo. A continuación, aludiremos brevemente a cada uno de ellos:

a) Las presunciones. Este medio probatorio consiente al juez dar por acreditado el nexo causal $^{19}$, aun cuando no exista prueba directa en torno a su configuración, pues justamente ese es su objetivo: suplir la deficiencia de prueba directa respecto de un determinado hecho ${ }^{20}$. Esta ha sido precisamente la solución adoptada por la Corte Suprema en algunos casos en que no existe total claridad sobre si el fallecido escuchó el anuncio radial del Intendente descartando el peligro de tsunami. En estos casos, sobre la base de lo que normalmente hace una persona en esas circunstancias, la Corte tuvo como hecho de la causa que las víctimas sí escucharon la transmisión radial en cuestión $^{21}$. Sin embargo, esta solución no es generalizable, pues la presunción requiere de "antecedentes o circunstancias conocidas" 22 que le sirvan de base, que no siempre obran en el proceso.

b) Los estándares probatorios. Estos mecanismos, cuyo punto de partida es asumir que la "verdad" es inalcanzable ${ }^{23}$, indican al juez, en términos probabilísticos, el grado

recurso a presunciones; o bien mediante artificios -como la pérdida de chance- que permiten una repartición de los riesgos de una determinada actividad entre las partes involucradas. Cfr. Nocco, 2006, p. 1243. En sentido similar, Múrtula, 2006, pp. 18-19.

${ }^{18}$ En este sentido, específicamente a propósito de la responsabilidad proporcional, MARTín-CaSALS, 2013 , pp. 318 ss.

${ }^{19}$ Hunter, 2015, pp. 209 ss.

${ }^{20}$ CORDOPATRI, pp. 274 ss.

${ }^{21}$ V. gr. Rol No 1629-2013, un análisis de este caso puede verse en SAN Martín, 2013, pp. 297-308 y en Lara y García-Huidobro, 2014, pp. 163-194.

${ }^{22}$ Según el artículo 47 C.C. "Se dice presumirse el hecho que se deduce de ciertos antecedentes o circunstancias conocidas".

${ }^{23}$ Pues, "conceptualmente cabe la posibilidad de que un enunciado esté probado y que, contemporáneamente, sea falso”. Ferrer, 2007, p. 20. Véase también Ferrer, 2002, p. 1 ss.; Taruffo, 2011, pp. 48 ss.; PAPAYANNIS, 2014, pp. 113 ss. 
de convicción necesario para tener por probado un hecho ${ }^{24}$. El grado de probabilidad suficiente no es pacífico ni unívoco en los distintos sistemas; sin embargo, tratándose de la responsabilidad civil, puede afirmarse que en los ordenamientos del Common Law la probabilidad se ha fijado en $50 \%$ más uno de probabilidades (more likely than not o more probable than not), mientras que en los de tradición continental es variable y va desde la probabilidad cercana a la certeza, al "más probable que no" ${ }^{25}$. Ahora bien, ciertamente los problemas probatorios que enfrentan las víctimas de daños dependen del estándar vigente en su ordenamiento. Con todo, la regla de base es siempre la misma: existe nexo de causalidad entre el hecho del agente y el daño, toda vez que, removido mentalmente el primero, desaparece el segundo, pero no es necesaria la absoluta certeza en tal sentido, basta con que se alcance el estándar de convicción exigido. De esta manera, en lo que aquí respecta, es posible sostener que los estándares probatorios menos exigentes (50\% más uno) funcionan de facto como una regla de facilitación de la prueba del nexo causal en contextos de elevada incertidumbre, como son los casos de desastres naturales. Con todo, los estándares probatorios vigentes en un ordenamiento responden a cuestiones de política jurídica, por tanto, resulta cuestionable que ellos puedan ser diferenciados para distintas tipologías de daños, al contrario, en virtud del principio de igualdad, parece que lo correcto es que sean uniformes para todas las víctimas. Con ello, en nuestro caso, la pregunta correcta sería cuál es el estándar probatorio que rige en Chile, cuestión que, por no existir claridad al respecto, excede con mucho los límites de este trabajo.

c) La "responsabilidad proporcional". Esta teoría propone que se obligue al demandado a indemnizar el daño en un porcentaje equivalente a la probabilidad de que él lo haya causado $^{26}$. Se trata de una doctrina particularmente popular a nivel doctrinario en el Common $\mathrm{Law}^{27}$, pero también ha entrado en la órbita del sistema continental ${ }^{28}$. En el contexto hispánico, una ferviente defensa de sus bondades ha sido planteada por Medina Alcoz, quien la destaca por sobre los sistemas de probabilidad preponderante, de la inversión legal de carga de la prueba, así como del estándar probabilístico especial (suave o riguroso) ${ }^{29}$. En Chile, sin embargo, no encontramos por el momento estudiosos que hayan propuesto este sistema como una fórmula general, aunque sí es posible observar una de sus variantes: la pérdida de oportunidad.

${ }^{24}$ Por todos, Larroucau, 2012, pp. 783-808.

25 Acerca del particular, vid Larroucau, 2012, pp. 783-808; Barros, 2006, pp. 378 ss.; Nocco, 2006, pp. 1238-1245; TAruffo, 2008, pp. 251 ss.; Cooke y Cowling, 2005, pp. 251-256; Cooke, 2009, pp. 67-68; PAPAyannis, 2014, pp. 100 ss.

${ }^{26}$ Respecto del punto Martín-Casals, 2016, pp. 43-66; Koch, 2016, pp. 67-86.

${ }^{27}$ Vid WeIr, 2004, pp. 511-552; Green (Michael) 2005 pp. 352-400; GreEN (Sarah), 2005, pp. 159175; Leshem y MiLler, 2009, pp. 345-382.

${ }^{28}$ Vid Gilead, et alt., 2013; Koch, 2016, pp. 67-86; Martín-Casals, 2016, pp. 43-66.

${ }^{29}$ Cfr. Medina, 2018, p. 84. 
d) La pérdida de oportunidad ${ }^{30}$. Esta solución consiste en otorgar a la víctima que no logra satisfacer el estándar probatorio del nexo de causalidad una indemnización limitada, existiendo dos alternativas para la determinación del quantum indemnizatorio. En la primera, se valora la chance en sí misma, considerando su pérdida como un daño autónomo. En la segunda, se indemniza un porcentaje del daño real sufrido (esto es la frustración de la ventaja que esperaba obtener), conforme con las probabilidades de que efectivamente hubiese obtenido dicha ventaja. En todo caso, en ambas versiones la teoría constituye un paliativo a las dificultades probatorias que enfrenta la víctima, pues si ella pudiera satisfacer el estándar probatorio exigido tendría derecho a la indemnización integral del daño. Asimismo, aunque no siempre se diga expresamente, la pérdida de la oportunidad se presenta como una especie de responsabilidad proporcional ${ }^{31}$ en "favor de la víctima"32, quien, aunque no logre acreditar el nexo causal, obtendrá alguna indemnización. Es admisible señalar que la jurisprudencia chilena de los últimos años ha hecho copioso uso de esta teoría para resolver problemas de responsabilidad médica, pero también en casos relativos al 27F, identificando la pérdida de chance con la "pérdida de la posibilidad de luchar dignamente por su vida" ${ }^{3}$. Aunque en estos fallos resulta altamente cuestionable el razonamiento subyacente, por diferentes motivos: en primer lugar, porque no es claro que los fallecidos hayan tenido una real oportunidad de ponerse a salvo del tsunami, en segundo lugar, porque no queda claro bajo cuál variante se realiza la determinación del quantum indemnizatorio ${ }^{34} \mathrm{y}$, finalmente, porque hacen derivar de la pérdida de la oportunidad supuestamente padecida por los fallecidos un daño moral para los dolientes, configurando en la práctica un nexo de causalidad directo entre la "falta de servicio" y el daño moral sufrido por las víctimas por repercusión. Aunque de forma intuitiva, es posible aceptar que las soluciones contenidas en estas sentencias responden a la conmoción pública que ha generado el $27 \mathrm{~F}$ y al hecho de que se demanda por muerte, por lo que no necesariamente constituyen jurisprudencia asentada en la materia, quedando así abierto el problema de cómo lidiar con la incertidumbre causal en casos de desastres naturales.

e) La teoría del aumento del riesgo. Esta doctrina postula que, incluso cuando no sea del todo claro que efectivamente el daño ha sido producto del actuar del agente, a este se le obligue a indemnizar por el hecho de que él aumentó el riesgo de que la víctima sufriera un daño ${ }^{35}$. Existiendo también dos variantes en cuanto al monto de la indem-

${ }^{30}$ Acerca de esta doctrina, por todos, vid Ríos y Silva, 2014, pp. 15 ss.

31 En este mismo sentido: Tassone, 2007, p. 1108; Medina, 2018, passim. En sentido similar, a propósito de la situación en Francia, Moréteau, 2013, pp. 143 y 144.

32 Acerca de esta materia, vid Gilead et alt., 2013, pp. 39 ss.

33 V. gr. Corte Suprema, 17.04.2018, rol 18225-2017; 16.11.2017, rol 4658-2017; у 17.01.2018, rol 5094-2017.

${ }^{34}$ Esta mezcla se advierte en el hecho de que, por un lado, establecen que la pérdida de chance permite lidiar con la incerteza causal del caso y, por el otro, atribuyen una indemnización valorando el daño moral padecido por las víctimas por rebote, como si indemnizaran un daño autónomo.

${ }^{35}$ Respecto de este tema, vid Green (Sarah), 2005, pp. 161-162. 
nización: resarcimiento integral o proporcional al incremento del riesgo. Esta segunda variante ha sido recogida de manera excepcional en Inglaterra, bajo dos requisitos: incertidumbre científica acerca de la causa del daño y que esté establecido que ese daño es el resultado del mismo (o al menos similar) riesgo al que fue expuesta la víctima ${ }^{36}$. Sin embargo, se ha puesto en evidencia que esta forma de razonamiento es difícilmente distinguible de la pérdida de chance ${ }^{37}$ y, por lo mismo, la doctrina más reciente las estudia conjuntamente como variantes de la "responsabilidad probabilística"38.

B. Necesaria concurrencia entre condiciones humanas y naturales: problemas en relación con el principio de resarcimiento integral del daño

Hasta ahora hemos abordado el problema del nexo causal desde una perspectiva binaria: hay o no hay nexo de causalidad. Sin embargo, es posible configurar una tercera alternativa, esto es: si bien el fenómeno natural era apto para producir el daño, su magnitud se vio agravada por la concurrencia de la conducta humana. Más allá de los evidentes problemas probatorios, esta alternativa lleva a preguntarse acerca de la posibilidad de establecer un concurso causal entre el fenómeno natural y la conducta del demandado. A esto me referiré en seguida.

Para entender este punto lo primero que es posible señalar es que jurídicamente el desastre natural no constituye una defensa en sí mismo, sino el presupuesto fáctico de la ocurrencia de un caso fortuito o fuerza mayor, de ahí que la disciplina de los desastres esté regida por las conclusiones generales aplicables a esta institución clásica del derecho privado ${ }^{39}$, cuya revisión se hace necesaria para el correcto encuadramiento de los desastres naturales en el sistema de responsabilidad civil ${ }^{40}$. Esta necesidad deriva de la circunstancia ya explicitada de que los desastres naturales tienen como factor desencadenante un fenómeno natural totalmente independiente de la voluntad humana y, por esta razón, de suyo inevitable. La cuestión, entonces, es si esa circunstancia fáctica tiene alguna repercusión jurídica a efectos de establecer la cuantía indemnizatoria; en otras palabras, si es posible configurar un concurso entre condiciones humanas y naturales ${ }^{41}$. En Chile, en general, esta pregunta está ausente de la literatura especializada, pues parte de la base del efecto "liberador" del caso fortuito, en consecuencia, se plantea solo como una causal de exención total de responsabilidad ${ }^{42}$.

\footnotetext{
36 Oliphant, 2013, p. 128.

37 Oliphant, 2013, p. 133.

38 STEEL, 2015, pp. 290 ss.

39 De esta materia, vid TApia, 2012, pp. 57 ss.; Veloso, 2012, pp. 63 ss.
}

${ }^{40}$ Desde luego, esta revisión puede tener consecuencias que vayan más allá de los desastres naturales, generalizándose para cualquier tipo de caso fortuito. Lo importante aquí es que ella se hace absolutamente necesaria cuando se trata de este tipo de hechos dañinos.

${ }^{41}$ Exactamente en estos términos plantea la cuestión RoDRÍGUEZ GREZ, quien se inclina por la respuesta negativa, vid RoDríGuEz, 2015, pp. 448 ss.

42 Vid Alessandri, 1943, p. 602; Coustasse e Iturra, 1958, pp. 129 ss.; Fueyo, 2004, p. 410; Ramos, 2008, p. 267; Troncoso, 2011, p. 196; Brantt, 2010, pp. 161 ss.; Brantt, 2011, pp. 70 ss.; TApia, 2013, p. 119; Abeliuk, 2014, t. II, p. 975; Meza, 2014, p. 122; Barrientos, 2016, t. I, pp. 200 ss. 
En efecto, la fuerza mayor ha sido entendida tradicionalmente como una defensa "todo o nada”, como causa de exoneración total de responsabilidad. En este sentido, afirma Barros: "la fuerza mayor comprende hechos ajenos al demandado (...) En virtud del principio de la causa necesaria, la responsabilidad no exige que el ilícito haya sido la única causa del daño resultante, sino que basta con que haya tenido una influencia significativa en su producción. Lo usual, por lo demás, es que los accidentes se produzcan por la concurrencia de la culpa y de innumerables circunstancias, de estas el derecho hace abstracción para efectos de establecer la responsabilidad" 43 .

Sin embargo, la defensa "todo o nada" no ha sido absoluta en todos los tiempos ni en todos los ordenamientos jurídicos. Así, en Francia, a raíz del caso Lamoricièrem, decidido en los años cincuenta, tuvo lugar la llamada "causalidad parcial", que daba lugar a la repartición de la responsabilidad entre las múltiples causas de un mismo daño, fueran estas humanas o naturales ${ }^{44}$. Esta doctrina fue abandonada ${ }^{45}$, pero en los últimos años está siendo revalorizada y es posible encontrar autores que abogan por su reposición ${ }^{46}$. En Italia ha tomado fuerza la idea de responsabilità parziaria ${ }^{47}$, y desde el 2009 se viene discutiendo acerca de la posible concurrencia entre causas humanas y naturales ${ }^{48}$. El fundamento base de la responsabilidad parcial es que el agente no debe ser obligado a indemnizar más que aquella parte de daño que su hecho efectivamente contribuyó a producir. En Chile, aunque sin mayores profundizaciones, esta idea ha sido propuesta por Baraona, a propósito del incendio de un bosque al que contribuyeron varios "factores", pero la Corte condenó íntegramente al dueño del predio vecino ${ }^{49}$. También la sugiere Corral, para quien "si en algún supuesto cabe imaginar que caso fortuito y comportamiento negligente actúan como concausas en forma necesaria y simultáneamente, quizá lo más justo sería no absolver totalmente de responsabilidad al agente, pero sí reducir el monto de la indemnización de manera proporcional a la entidad del aporte causal del agente" ${ }^{50}$. A su vez, Barros, luego de haber afirmado que la "fuerza mayor comprende hechos ajenos al demandado", se cuestiona que la indemnización deba fijarse con un criterio todo o nada, pues, afirma, "puede ocurrir que la concurrencia de culpa y caso fortuito sean determinantes en la producción del primer daño, de modo que no parezca justo atribuir el total de los daños al hecho culpable o al revés" ${ }^{2}$. Si bien el autor es escéptico respecto de la solución, lo relevante es que pone de manifiesto precisamente lo que queremos destacar: los fenómenos naturales a la base de los desastres naturales pueden revestir caracteres tales que admite cuestionarse la aplicación de una regla que, o bien deje

${ }^{43}$ BArros, 2006, p. 415.

${ }^{44}$ Chabas, 1967 , pp. 135 ss.

45 Chabas, 2009, pp. 73-74.

46 Vid QuÉzel-AmbrunaZ, 2010, pp. 297 ss.

47 Violante, 2004, pp. 7 ss.; TASSONE, 2007, pp. 1095-1109.

48 CApecchi, 2010, pp. 372-387; Nocco, 2012, pp. 149-164.

49 Para el autor, "la concausa fue un elemento que debió considerarse para moderar los daños". BARAONA, 2003, p. 366.

${ }^{50}$ Corral, 2013, p. 193. En el mismo sentido se pronuncia en Corral, 2010, p. 465.

${ }^{51}$ Barros, 2006, p. 415. 
a la víctima sin indemnización alguna, o bien le imponga al demandado una obligación de indemnizar incluso el daño que la víctima habría sufrido aun sin su intervención.

La idea de que el agente no debe responder sino del daño que su hecho ha contribuido a causar aparece intuitivamente justa. Sin embargo, no es tan simple, pues podría llegarse al absurdo de tener que establecer tantas parcelas indemnizatorias como condiciones han concurrido al daño. En consecuencia, es necesario identificar un criterio jurídico que permita atribuir carácter de concausa al fenómeno natural que desencadena el desastre. En principio, podría decirse que tal criterio corresponde al "carácter extraordinario" del evento; en efecto, la doctrina especializada afirma que "la catástrofe es el efecto perturbador que provoca sobre un territorio un episodio natural de rango extraordinario y que a menudo supone la pérdida de vidas humanas" ${ }^{2}$. Asimismo, esto supone determinar qué se entiende por "extraordinario" y, sobre todo, en qué se diferenciaría la extraordinariedad, de la imprevisibilidad propia del caso fortuito, máxime cuando, como reconoce la doctrina, "los fenómenos de la naturaleza, en términos generales, son típicamente sucesos imprevisibles, ya que su ocurrencia no es posible razonablemente de prever" 53 .

Como se aprecia, los desastres naturales, en cuanto especiales hipótesis de daños, obligan a repensar categorías que parecían establecidas, como la regla todo o nada en relación con los efectos del caso fortuito, y abrirse a la posibilidad de aceptar una responsabilidad parcial, que acepte la concurrencia entre causas humanas y naturales. Llegar a una conclusión respecto de esta materia constituye otro de los desafíos que presenta esta materia.

\section{Los desastres naturales como hipótesis de daño contingente}

\section{A. Los desastres naturales como daños (en gran medida) evitables}

Todas las naciones están expuestas, en mayor o menor medida, a eventos naturales extremos, potencialmente dañinos, y conocidos en este contexto como "amenazas". Sin embargo, estos fenómenos no siempre provocan desastres. "El riesgo de desastres surge cuando las amenazas/peligros interactúan con factores de vulnerabilidad físicos, sociales, económicos y ambientales" 54 . La "vulnerabilidad" es una condición previa-que se manifiesta durante el desastre- y al mismo tiempo es un indicador de la exposición del capital y de la capacidad de tolerancia y resiliencia al daño por parte de personas, hogares, comunidades y países ${ }^{55}$.

Debido a que los fenómenos naturales no generan desastres, sino que estos dependen de la vulnerabilidad, se aprecia la necesidad de establecer políticas de prevención, mediante el manejo y gestión de las condiciones de vulnerabilidad ${ }^{56}$. Al efecto, los expertos en gestión de riesgos de desastres la dividen en dos etapas, dependiendo del punto de referencia

\footnotetext{
52 Olcina, 2006, p. 65.

53 Veloso, 2012, p. 65.

${ }^{54}$ Marco de Acción de Hyogo para 2005-2015, p. 2.

55 CEAL, 2014. Vid también AaVv, 2015; TheInternational Bank for Reconstruction and Development/The World Bank, 2011.

${ }^{56}$ Nivolianitou, 2002, pp. 161-174; Doyle et al. pp. 75-101, 2014; Ríos, 2010, pp. 27-43.
} 
temporal en relación con el desastre: la gestión correctiva o compensatoria y la prospectiva. La primera alude al manejo de la emergencia y a la recuperación ${ }^{57}$, mientras que "la gestión prospectiva hace referencia a la gestión de riesgos futuros, los cuales buscan normar y/o controlar nuevos desarrollos mediante la planificación e inversión pública, específicamente con herramientas como el ordenamiento territorial, la planificación del desarrollo, la planificación sectorial y planificación financiera, las cuales se proyectan a futuro para la evitar la creación o recreación de nuevos riesgos" 58 .

Se pone así en evidencia la necesidad de actuar no solo expost, sino principalmente ex ante, minimizando el riesgo de ocurrencia de desastres, especialmente mediante la ordenación del territorio afectado por la amenaza ${ }^{59}$, lo que exige tener en consideración la interacción público-privada que provoca el desastre ${ }^{60}$. De esta manera, los particulares pueden y deben adoptar medidas para evitar ser víctimas o causantes de desastres.

En este punto, cobra importancia la responsabilidad civil como mecanismo de prevención de daños. Especialmente relevante será la acción por daño contingente consagrada en el artículo 2333 del Código Civil, aunque, como se dirá, las condiciones de procedencia de esta acción en relación con los desastres naturales revisten características particulares, que presentan desafíos a tener en cuenta por los estudiosos de la disciplina. La puesta en evidencia de estos desafíos es el objetivo de esta parte del texto.

B. La acción de daño contingente como herramienta de prevención de desastres naturales

En Chile existe una herramienta pionera en la función preventiva de la responsabilidad, que permite evitar efectivamente ciertos daños: la "acción de daño contingente" del artículo 2333 CC. Con algunas excepciones ${ }^{61}$, la doctrina no ha dedicado mayor atención a esta norma. No obstante, es posible destacar algunos aspectos relevantes que se deben considerar a la hora de intentar la aplicación de la regla a la prevención de desastres naturales.

El daño contingente puede ser conceptualizado como aquel que no se ha verificado, pero respecto del que existen razonables probabilidades de ocurrencia si no se remueve la fuente de peligro. Esto supone un problema para los daños derivados de desastres naturales, pues no es posible anticipar circunstanciadamente el fenómeno natural desencadenante, lo que conlleva la necesidad de identificar criterios que permitan concluir bajo qué condiciones existe una "razonable probabilidad" de ocurrencia de un desastre natural, habida cuenta de la escasa capacidad de predecir los fenómenos que los desencadenan. En efecto, tratándose de fenómenos naturales, la determinación de la probabilidad de ocurrencia supone tener en

${ }^{57}$ Camus et al., 2016, p. 12.

58 Cfr. Camus et al., 2016, p. 12.

59 Cfr. Ayala-Carcedo et al., 2006, p. 47.

${ }^{60}$ Vid Ríos, 2010, p. 30.

${ }^{61}$ Como excepciones vid Molinari, 2004, 201 ss.; Díez y Delgado, 2005, pp. 144-150; Díez, 2005, pp. 317-321; DiEZ, 2016a, pp. 133-153; DíEZ, 2016b, pp. 257-286. 
cuenta la evidencia científica al respecto ${ }^{62}$, y, aunque en términos generales la predicción de los fenómenos naturales a largo plazo es posible, la comunidad científica reconoce que la predicción a corto y mediano plazo no siempre lo es, especialmente tratándose de tsunamis, terremotos y erupciones volcánicas, que son justamente las principales preocupaciones en Chile ${ }^{63}$.

Asimismo, un desafío para los estudiosos de la disciplina consiste en identificar la evidencia científica relevante a efectos de adoptar medidas de prevención de los desastres y, sobre todo, establecer sobre qué base se van a calcular las "razonables probabilidades": sobre una base estadística, que tenga en cuenta los anteriores desastres ocurridos en la zona, o bien sobre un cálculo meramente probabilístico, de acuerdo con las condiciones de la zona estudiada. Ambos aspectos resultan problemáticos, pues, por un lado, puede no haber registros de un determinado tipo de desastre en la zona analizada y, por el otro, pueden existir las condiciones de amenaza que indiquen la posibilidad de su ocurrencia. Un ejemplo de esta dicotomía es una falla geológica de la que no existen registros del último terremoto que se generó en ella.

Otro aspecto para destacar en esta sede es la legitimidad activa en relación con el daño contingente. En efecto, según el artículo 2333, cuando el daño amenace a "persona indeterminada" la acción es popular. Sin embargo, no queda claro si debe tratarse de una persona posiblemente afectada o puede ser, por ejemplo, una ONG internacional interesada en la prevención de un determinado desastre. La respuesta a esta pregunta pasa por establecer cómo se aplica la noción de acción popular desarrollada por la doctrina a los desastres naturales.

A su vez, también resulta discutible cómo se determina al legitimado pasivo de la acción. Según el texto de la norma, el legitimado pasivo es aquel por cuya imprudencia o negligencia se genera la amenaza de daño, sin distinguir entre privados e instituciones estatales, con lo que es perfectamente posible que la acción se ejerza en contra de una entidad estatal ${ }^{64}$.

Así, resulta oportuno preguntarse si puede ser considerada negligencia la inactividad del Estado, que no adopta medidas para mitigar o eliminar las vulnerabilidades. Esta posibilidad se entronca directamente con el hecho de que, mientras más recursos se asigne a la eliminación de las vulnerabilidades, menor es el riesgo de desastre. Está demostrado que entre más económicamente desarrollado es el país (por tanto invierte mayor cantidad de recursos en prevención), menor potencial dañino tienen los fenómenos naturales, ya sea por su mayor tolerancia o bien su mayor índice de resiliencia ${ }^{65}$. Sin embargo, no puede obviarse el hecho de que obligar a la administración a adoptar medidas preventivas de desastres, por vía judicial, atenta contra la discrecionalidad presupuestaria, pues, en la medida que los particulares demanden civilmente la realización de ese tipo de actividades, el Estado se verá constreñido a invertir en ellas, aunque no las haya presupuestado, atentando así contra la

\footnotetext{
62 Weiss, 2003, pp. 25-46; Taruffo, 2005, pp. 1285-1312, Taruffo, 2011, pp. 135-186.

63 Ayala-Carcedo y González, 2006, p. 34.

${ }^{64}$ En efecto, una de las pocas aplicaciones jurisprudenciales de la norma ha sido precisamente en contra de una entidad estatal, el Serviu. Los detalles del caso pueden verse en DíEz, 2005, pp. 317-321.

${ }^{65}$ CEPAL, 2014, p. 19.
} 
llamada discrecionalidad presupuestaria ${ }^{66}$. Por tanto, para establecer la legitimación pasiva del Estado en este tipo de casos es necesario determinar las condiciones de exigibilidad de las medidas que pueden impetrar los particulares, esto es, bajo qué condiciones el Estado estará obligado a actuar, sin que pueda invocar su discrecionalidad presupuestaria, asumiendo que este es un límite a la intervención judicial del Estado.

\section{OBSERVACiOnes finALES}

Como observaciones conclusivas de este texto, es posible afirmar que la proliferación de demandas de responsabilidad civil por los daños sufridos a raíz de desastres naturales exige un esfuerzo sistematizador por parte de los cultores de la responsabilidad civil, en la medida que los fenómenos naturales constituyen especiales categorías de hechos lesivos y que, en ausencia de seguros, el mecanismo de la responsabilidad representa la forma en que las víctimas de los desastres obtienen indemnidad.

En esta sede, estos esfuerzos han ido dirigidos a poner de relieve los principales desafíos que conlleva el correcto entendimiento de la responsabilidad civil derivada de desastres naturales, comenzando por el hecho de que los desastres son el fruto de la convergencia entre amenazas y vulnerabilidades, lo que determina que el fenómeno natural que los produce no es en sí mismo constitutivo de desastre, sino que este se genera cuando existen condiciones de vulnerabilidad previas, que afloran con el desastre. A todo ello debe sumarse el hecho de que, para que haya lugar a responsabilidad civil, es necesario concluir que la vulnerabilidad es atribuible a un tercero, quien tenía el deber jurídico de removerla (culpa). A esto se añaden las dificultades asociadas a la prueba del nexo de causalidad natural, especialmente apremiantes en este tipo de casos. Esta incertidumbre causal generalmente estará determinada por las circunstancias que rodean al desastre, pero también puede deberse a la escasa evidencia respecto de la influencia que un comportamiento distinto hubiese tenido en la evitación del desastre, lo que conlleva los problemas propios de la prueba de un contrafáctico. En doctrina, especialmente extranjera, se han ensayado varios mecanismos aptos para enfrentar los problemas de incertidumbre causal y que eventualmente pueden aplicarse en este tipo de daños, pero queda pendiente establecer cuál de ellos se condice mejor con el sistema de responsabilidad civil chileno.

Siempre en relación con el nexo causal, es admisible preguntar si es posible la concurrencia causal entre el fenómeno natural y la culpa del tercero, abandonando así la llamada regla "todo o nada", para dar paso a una responsabilidad parcial, que obligue al demandado a indemnizar solo aquella parte que le resulte causalmente atribuible. De aceptarse esta posibilidad, es necesario establecer los criterios que permitirán distinguir un desastre natural que genera responsabilidad parcial de aquel que queda en el terreno del caso fortuito. De esta manera, en lo que respecta a este punto, el desafío pendiente es doble: (i) se admite o no la causalidad parcial; y (ii) bajo qué criterios podría aplicarse.

\footnotetext{
${ }^{66}$ Letelier, 2012b, pp. 11 ss.; Ye et al., 2016, pp. 123-137; Hu et al., 2016, pp. 138-150.
} 
Finalmente, resulta legítimo elucubrar acerca de la posibilidad de recurrir a la herramienta del daño contingente como un mecanismo para prevenir la ocurrencia de desastres; sin embargo, la real potencialidad de esta herramienta dependerá de qué se entienda por razonables probabilidades de ocurrencia de desastre, lo que implica un ulterior desafío para el correcto encuadramiento de la responsabilidad civil derivada de desastres naturales.

\section{BIBLIOGRAFÍA}

Abeliuk Manasevich, René, 2014: Las obligaciones, 6ª edición, Santiago: LegalPublishing-Thomson Reuters.

Alessandri Rodríguez, Arturo, 1943: De la responsabilidad extracontractual en el derecho civil chileno, Santiago: Imprenta Universitaria.

Ayala-Carcedo, F. J. y GonzÁlez Jiménez, Á., 2006: "Mitigación de desastres naturales en el mundo y desarrollo sostenible: una aproximación al análisis del riesgo, en F. J. Ayala-Carcedo, L. Olcina Cantos, L. Laín Huerta, y Á. González Jiménez, Á., Riesgos naturales y desarrollo sostenible. Impacto predicción y mitigación, Madrid: Instituto Geológico y Minero de España, pp. 19-46.

Ayala-Carcedo, F. J., Olcina Cantos y Vilaplana, J. M., 2006: "Impacto socioeconómico y estrategias de mitigación de los riesgos naturales en España en el período 1990-2000”, en F. J. Ayala-Carcedo, L. Olcina Cantos, L. Laín Huerta, y Á. González Jiménez, Á., Riesgos naturales y desarrollo sostenible. Impacto predicción y mitigación, Madrid: Instituto Geológico y Minero de España, pp. 47-62.

BARAONA GonZÁLEZ, Jorge 2003: "La causa del daño en la jurisprudencia reciente", Revista Chilena de Derecho, Vol. 30, $\mathrm{N}^{\circ} 2$.

Barros Bourie, Enrique, 2006: Tratado de responsabilidad extracontractual, Santiago: Editorial Jurídica de Chile.

Brantt Zumarán, María Graciela, 2010: El caso fortuito y su incidencia en el derecho de la responsabilidad civil contractual, Santiago: AbeledoPerrot.

Calabresi, Guido, 1970: The cost of accidents. A legal and economic analysis, New Haven-Lóndres: Yale University Press.

Calabresi, Guido, 1978: “Torts-The Law of the Mixed Society”, Texas Law Review, № 519.

Camus, P., Arenas, F., Lagos, M. y Romero, A., 2016: "Visión histórica de la respuesta a las amenazas naturales en Chile y oportunidades de gestión del riesgo de desastre", Revista de Geografía Norte Grande, No 64.

Cane, Peter, 2014: "Tort Law and Public Functions", en John Oberdiek, Philosophical Foundations of the Law of Torts, Oxford: Oxford University Press, pp. 148-168.

CAPECCHI, Marco, 2010: "Il reveriment della Corte di Cassazione sulla interpretazione dell'art. 2055 C.C.", Danno e Responsabilità, No 4 .

CEPAL, 2014: Manual para la evaluación de desastres, disponible en: https://repositorio.cepal.org/ bitstream/handle/11362/35894/1/S2013806_es.pdf [Fecha de consulta: 15.12.2018]

CHABAs, François, 1967: L'influence de la pluaralitè des causes sur le droite a réparation, Paris: L.G.D.J.

Chabas, François, 2009: Cien años de responsabilidad civil en Francia, Santiago: Flandes Indiano.

Cooke, Peter 2009: "Clarification and corrections to 'On the attribution of probabilities to the causes of disease' by Peter Cooke and Arianna Cowling”, Law, Probability and Risk, $\mathrm{N}^{\circ} 8$.

Cooke, Peter, Cowling, Arianna 2005: "On the attribution of probabilities to the causes of disease", Law, Probability and Risk, $\mathrm{N}^{\circ} 4$.

Cordopatri, Franco, 1986: Voz presunzione (dir. proc. civ), en Enciclopedia del Diritto, vol. XXXV, Milán: Giuffrè. 
Corral Talciani, Hernán, 2010: "Responsabilidad civil en la construcción de viviendas. Reflexiones sobre los regímenes legales aplicables a los daños provocados por el terremoto del 27 de febrero de 2010", Revista Chilena de Derecho, vol. 37 No 3 .

Corral TAlCiani, Hernán, 2013: Lecciones de responsabilidad civil extracontractual, $2^{a}$ edición, Santiago: LegalPublishing-Thomson Reuters.

Coustasse Del C., Alberto y IturRa A., Fernando, 1958: El caso fortuito ante el Derecho civil, Santiago: Editorial Jurídica de Chile.

Díez Schwerter, José Luis y Delgado Schneider, Verónica, 2005: “Algunas útiles herramientas olvidadas en nuestra práctica del Derecho de Daños, Revista de Derecho Universidad de Concepción, $\mathrm{N}^{\circ} 214$ (2003).

Díez Schwerter, José Luis, 2005: "Notas sobre la acción preventiva de daños del artículo 2333 del Código Civil", Revista de Derecho Universidad de Concepción, pp. 217-218.

Díez Schwerter, José Luis, 2016a: "La acción de dañó contingente del artículo 233 del Código Civil: sus elementos y ámbito de aplicación”, Revista de Derecho PUCV (Valparaíso), No 46.

Díez Schwerter, José Luis, 2016b: "La aplicación de la acción por daño contingente en Chile, Colombia y Ecuador: del modelo de Bello a nuestros días", Revista de Derecho Privado, Universidad del Externado (Bogotá), № 30.

Domínguez Águila, Ramón, 1966: "El hecho de la víctima como causal de exoneración de la responsabilidad civil”, Revista de Derecho, Universidad de Concepción, N 136.

Doyle, Emma, Mc Clureb, John, Patonc, Douglas, Johnstonad, David M., 2014: "Uncertainty and decision making: Volcanic crisis scenarios", International Journal of Disaster Risk Reduction, $\mathrm{N}^{\circ} 10$.

Faure, Michael y Hartulef, Ton, 2006: Financial Compensation for Victims of Catastrophes: A Comparative Legal Approach, Viena: Springer.

Faure, Michael y Jing, Liu, 2012: "The Tsunami of March 2011 and the Subsequent Nuclear Incident at Fukushima: Who Compensates the Victims?”, William \& Mary Environmental Law and Policy Review, Vol. 37, No 129.

FERNÁNDEZ RichaRd, José, 2011: "Los eventos catastróficos y en especial el terremoto frente al derecho: la responsabilidad de los órganos administrativos", Revista de Derecho Público, No 74.

Ferrer Beltrán, Jordi, 2002: Prueba y verdad en el Derecho, (2ª edición), Madrid: Marcial Pons.

Ferrer Beltrán, Jordi, 2007: La valoración racional de la prueba, Madrid: Marcial Pons.

GatTI, Stefano, 2015: "Concorso di cause e principio di proporzionalità", Danno e Responsabilità, No 6.

Gilead, Israel, Green, Michael D. y KoCH, Bernhard A., 2013: General Report, en Israel GileAD, Michael D. Green y Bernhard A KocH (eds.). Proportional Liability: Analitical and Comparative Perspectives. Berlín - Boston: De Gruyter.

Gilead, Israel, Green, Michel D., Koch, Bernhard A. (editores), 2013: Proportional Liability: Analytical and Comparative Perspectives, Tort and Insurance Law, Vol. 33, Berlin: Boston De Gruyter.

GNANI, Alessandro, 2013: "Causa umana e causa naturale in concorso: nuovi possibili scenari dopo le sentenze della Cassazione", Danno e Responsabilità, $\mathrm{N}^{\circ} 11$.

Green, Michael D., 2005: "The Future of Proportional Liability”, en Stuart, Madden (editor), Exploring tort law, Cambridge: Cambridge University Press.

GreEN, Sarah, 2005: “The risk pricing principle: a pragmatic approach to causation and apportionment of damages", Law, Probability and Risk, $\mathrm{N}^{\circ} 4$.

Hunter Ampuero, Iván, 2015: "Las dificultades probatorias en el proceso civil. Tratamiento doctrinal y jurisprudencial, críticas y una propuesta". Revista de Derecho, Universidad Católica del Norte, Año 22, $\mathrm{N}^{\circ} 1$.

Infante Ruiz, Francisco José, 2002: La responsabilidad por daños. Nexo de causalidad y "casos hipotéticos", Valencia, Tirant. 
Jaramillo Contreras, Marcos, 2012: "La regulación jurídica en torno a los desastres en Japón”, en Derecho y catástrofe: lecciones del terremoto, Santiago: Facultad de Derecho Universidad de Chile, pp. 11-24.

Jordano Fraga, Jesús, 2000: La reparación de los daños catastróficos, Madrid: Marcial Pons.

KосH, Bernhard A., 2016: "Proportional liability for causal uncertainty. How to works on the basis of a 200-year-old code”, en Miquel Martín-Casals, Diego M. Papayannis (editores), Uncertain causation in tort Law, Cambridge: Cambridge University Press, pp. 67-86.

Kousky, Carolyn y Cooke, Roger, 2012: "Explaining the Failure to Insure Catastrophic Risks", The Geneva Papers, pp. 206-227.

LACroiX. Caroline, 2008: La réparation des dommages en cas de catastrophes, Paris: L.G.D.J.

Lara Arroyo, José Luis, García-Huidobro Herrera, Luis Eugenio, 2014: "27F: ¿Terremoto en la responsabilidad del Estado?, Anuario de Doctrina y Jurisprudencia. Sentencias destacadas 2013, Santiago: Ediciones Libertad y Desarrollo, pp. 163-194.

Larroucau, Torres, Jorge, 2012: "Hacia un estándar de prueba civil", Revista chilena de Derecho, Vol. 39, $\mathrm{N}^{\circ} 3$.

Larroucau, Torres, Jorge, 2014: “¿Cómo se prueba la responsabilidad civil médica en la justicia chilena"?, Revista de Derecho (Valdivia), Vol. XXVII, No 2.

Leshem Shmuel, Miller, Geoffrey P., 2009: "All-or-Nothing versus Proportionate Damages", Journal of Legal Studies, Universidad de Chicago, Vol. 38, pp. 345-382.

LETELIER WARTENBERG, Raúl, 2001: La fuerza mayor en la responsabilidad extracontractual de la administración del Estado, Santiago: Jurídica Conosur.

LETELier WARTENBERg, Raúl, 2012a: "Falta de servicio en situación de catástrofes naturales", en Letelier, Raúl (coordinador), La falta de servicio, Santiago: Thomson Reuters, pp. 303-321.

Letelier Wartenberg, Raúl, 2012b: "La falta de servicio. Aciertos y desafíos pendientes", en Letelier, Raúl (coordinador), La falta de servicio, Santiago: Thomson Reuters, pp. 1-26.

Martín-Casals, Miquel, 2013: "Causal uncertainty and Proportional Liability in Spain", en Israel Gilead, Michael D. Green y Bernhard A. Koch (editores). Proportional Liability: Analitical and Comparative Perspectives. Berlín - Boston: De Gruyter.

Martín-Casals, Miquel, 2016: "Proportional liability in Spain. A bridge too far?”, en Miquel Martín-Casals y Diego M. Papayannis (editores), Uncertain causation in tort Law, Cambridge: Cambridge University Press, pp. 43-66.

Medina Alcoz, Luis, 2018: La responsabilidad proporcional como solución a la incertidumbre causal, Madrid: Civitas-Thomson Reuters.

Molinari VAldés, Aldo, 2004: De la responsabilidad civil al derecho de daños y tutela preventiva civil, Santiago: LexisNexis.

Moréteau, Olivier, 2013: "Causal Uncertainty and Proportional Liability in France", en Israel Gilead, Michael D. Green y Bernhard A. Koch (editores). Proportional Liability: Analitical and Comparative Perspectives. Berlín - Boston: De Gruyter.

Múrtula Lafuente, Virginia, 2006: "Causalidad alternativa e indeterminación del causante del daño en la responsabilidad civil”, en InDret, 2/2006. Disponible en: http://www.indret.com/ pdf/351_es.pdf [Fecha de consulta: 31.01.2019].

Nivolianitou, Zoe, 2002: "Risk analysis and risk management: a European insight", Law, Probability and Risk, $\mathrm{N}^{\circ} 1$.

Nocco, Luca, 2006: "Causalità: dalla probabilità logica (nuovamente) alla probabilità statistica, la Cassazione civile fa retromarcia”, Danno e Responsabilità, $\mathrm{N}^{\circ} 12$.

Nocco, Luca, 2012: "Rilevanza delle concause naturali e responsabilità proporzionale: un discutibile revirement della Cassazione”, Danno e Responsabilità, № 2 .

Olcina Cantos, J., 2006: "La ordenación del territorio en la mitigación de riesgos naturales en España: estudios de casos”, en F. J. Ayala-Carcedo, L. Olcina Cantos, L. Laín Huerta, y 
Á. González Jiménez, Á., Riesgos naturales y desarrollo sostenible. Impacto predicción y mitigación, Madrid: Instituto Geológico y Minero de España, pp. 65-88.

Onetto, Mauricio, 2017: Temblores de tierra en el jardín del edén. Desastres, memoria e identidad. Chile, siglos XVI-XVIII, Santiago: Centro de Investigaciones Diego Barros Arana, Dibam.

Palacios Roa, Alfredo, 2016: Historia de los megaterremotos ocurridos en Chile entre 1647 y 1906, Valparaíso: Ediciones Universitarias de Valparaíso.

PiZarro Wilson, Carlos, 2010: "Daños en la construcción, fuerza mayor y terremotos", Revista de Derecho de la Pontificia Universidad Católica de Valparaíso, $\mathrm{N}^{\circ}$ XXXIV.

Poblete Espíndola, Gustavo, 2015: "La falta de servicio ante los daños generados por desastres naturales (Corte Suprema)", Revista de Derecho (Valdivia), Vol. 28, № 1.

Posner, Richard A., 2004: Catastrophe: Risk and Response, Nueva York: Oxford University Press.

Quézel-Ambrunaz, Cristophe, 2010: Essai sur la causalitè en droit de la responsabilité civile, París: Dalloz.

RAIKES, Jontathan y MCBEAN, Gordon, 2016: "Responsibility and liability in emergency management to natural disasters: A Canadian example", International Journal of Disaster Risk Reduction, $\mathrm{N}^{\circ} 16$.

Ramos Pazos, René, 2008: De las obligaciones, $3^{a}$ edición, Santiago: LegalPublishing.

RatTi, Giovanni Battista, 2014: "Los contrafácticos en el Derecho. Un inventario de problemas", en Papayannis, Diego M. (editor), Causalidad y atribución de responsabilidad, Madrid: Marcial Pons, pp. 77-101.

Ríos ERAzo, Ignacio Javier, 2017: Incerteza causal en la responsabilidad civil: Jurisprudencia terremoto del 27 de febrero de 2010, Tesis para optar al Grado de Magíster en Derecho con mención en Derecho Privado, Universidad de Chile. Disponible en: http://repositorio.uchile.cl/bitstream/handle/2250/150944/ An\%C3\% A1lisis-jurisprudencial-sobre-la-responsabilidad-civil-extracontractual-del-Estadopor-falta-de-servicio-derivada-del-terremoto-y-posterior-tsunami-del-27F.pdf? sequence $=1$ [Fecha de consulta: 1.12.2018].

Ríos ERAzo, Ignacio Javier, Silva GoÑI, Rodrigo Pascual, 2014: Responsabilidad civil por pérdida de la oportunidad, Santiago: Editorial Jurídica de Chile.

Ríos, Diego Martín, 2010: "Urbanización de áreas inundables, mediación técnica y riesgo de desastre: una mirada crítica sobre sus relaciones”, Revista de Geografía del Norte Grande, No 47.

Rodríguez Grez, Pablo, 2015: Responsabilidad extracontractual, $2^{\mathrm{a}}$ edición, Santiago: Editorial Jurídica de Chile.

San Martín Neira, Lilian C., 2013: "Responsabilidad del Estado por falta de servicio "Valenzuela Flores y otros con Fisco de Chile" (Rol No 1629-2013)", Roma e América, No 34.

Schopf Olea, Adrián, 2015: "El ámbito de protección de la responsabilidad extracontractual y los daños puramente patrimoniales", Estudios de Derecho Civil X, Santiago: Thomson Reuters.

Silva Avaria, Bárbara y RiQuelme Segovia, Alfredo, 2018: Una identidad terremoteada. Comunidad y territorio en el Chile de 1960, Santiago: Ediciones Universidad Alberto Hurtado.

Steel, Sandy 2015: Proof of Causation in Tort Law, Cambridge: Cambridge University Press.

TAPIA RodrígueZ, Mauricio, 2012: "Catástrofe y fuerza mayor a propósito del terremoto y maremoto de 27 de febrero de 2010", en Derecho y catástrofe: lecciones del terremoto, Santiago: Facultad de Derecho Universidad de Chile, pp. 57-62.

TAPIA RodríGuez, Mauricio, 2013: Caso fortuito en el derecho chileno, Santiago: LegalPublishing.

TARUfFo, Michele, 2005: "Conocimiento científico y estándares de prueba judicial”, Boletin Mexicano de Derecho Comparado (nueva serie), año XXXVIII, No 14, pp. 1285-1312.

TARUffo, Michele, 2008: "La prueba del nexo causal", en TARuffo, Michele, La prueba (trad. Laura Manríquez y Jordi Ferrer), Madrid: Marcial Pons, pp. 251-276.

TARuffo, Michele, 2011: "La prueba científica en el proceso civil", en Ferrer, Jordi et al. Estudios sobre prueba, México: Universidad Nacional Autónoma de México, pp. 135-186.

TARuffo, Michele, 2011: La prueba de los hechos, (4ta., edición) trad. Jordi Ferrer Beltrán, Madrid: Trotta. 
TAssone, Bruno, 2007: "Solidarietà e parziarietà nell'ordinamento italiano, un'analisi operazionale", Danno e Responsabilità, No 11, pp. 1095-1109.

The International Bank for Reconstruction and Development/The World Bank, 2011: Peligros naturales, desastres evitables: La economía de la prevención efectiva, Washington DC: Gondo. Disponible en: https://www.gfdrr.org/sites/default/files/publication/peligros-naturalesdesastres-evitables-2010.pdf [Fecha de consulta: 15.12.2018].

Ugarte, A.M., Salgado, M., y Fuster, X., 2014: "Emergencia de sujeto político y experiencia de acción colectiva en desastres socionaturales: Análisis de casos en Santiago, Constitución y Chaitén, Chile”, en Catalina Arteaga A., y Ricardo Tapia Z., (editores) Vulnerabilidades y desastres socionaturales. Experiencias recientes en Chile Santiago: Editorial Universitaria, pp. 117-130.

UNISDR, 2015: América del Sur. Enfoque para la gestión de riesgos de desastres. Recuperado 20.07.2017, https://www.unisdr.org/we/inform/publications/42140.

Veloso Valenzuela, Paulina, 2012: “Caso fortuito y catástrofes”, en Derecho y catástrofe: lecciones del terremoto, Santiago: Facultad de Derecho Universidad de Chile, pp. 63-67.

VIney, Geneviève, 2007: Tratado de derecho civil. Introducción a la responsabilidad (trad.) Fernando Montoy Mateus, Bogotá: Editorial Universidad Externado de Colombia.

Violante, Umberto, 2004: La responsabilità parziaria, Nápoles: Edizioni Scientifiche Italiane.

WeIr, Tony, 2004: “ALL or Nothing?”, Tulane Law Review, 2003-2004, vol. 78.

WeIss, Charles, 2003: "Expressing scientific uncertainty", Law, Probability, and Risk, $\mathrm{N}^{\circ} 2$.

ZorziT, Daniela, 2012: "Il problema del concorso di fattori naturali e condotte umane. Il nuovo orientamento della Cassazione", Danno e Responsabilità, $\mathrm{N}^{\circ} 5$.

Meza Barros, Ramón, 2104: Manuel de derecho civil: de las obligaciones, $10^{a}$ edición, Santiago: Editorial Jurídica de Chile.

Troncoso Larronde, Hernán, 2011: De las obligaciones, $7^{a}$ edición: Santiago: LegalPublishingThomson Reuters.

Brantt Zumarán, María Graciela, 2011: "El caso fortuito: concepto y función como límite de la responsabilidad contractual”, en De la Maza Gazmuri, Íñigo (coordinador), Cuadernos de Análisis Jurídico VII, Santiago: Ediciones Universidad Diego Portales.

Fueyo LANeri, Fernando, 2004: Cumplimiento e incumplimiento de las obligaciones, $3^{a}$ edición, actualizada por Gonzalo Figueroa Yáñez, Santiago: Editorial Jurídica de Chile.

Barrientos Grandon, Javier, 2016: El código civil su jurisprudencia e historia, Santiago: Thomson Reuters-La Ley.

\section{Jurisprudencia Citada}

CORTE Suprema, sentencia de fecha 14 de abril de 2018, rol 18225-2017.

CoRTe Suprema, sentencia de fecha 16 de noviembre de 2017, rol 4658-2017.

CORTE Suprema, sentencia de fecha 17 de enero de 2018, rol 5094-2017. 